\title{
Neural activity changes underlying the working memory deficit in alpha-CaMKII heterozygous knockout mice
}

\section{Naoki Matsuo ${ }^{1,2+}$, Nobuyuki Yamasaki ${ }^{3,4 t}$, Koji Ohira ${ }^{1,2}$, Keizo Takao ${ }^{1,2,3,5}$, Keiko Toyama ${ }^{1,2}$, Megumi Eguchi ${ }^{6}$, Shun Yamaguchi ${ }^{6}$ and Tsuyoshi Miyakawa ${ }^{1,2,3,5 *}$}

\author{
1 Division of Systems Medical Science, Institute for Comprehensive Medical Science, Fujita Health University, Toyoake, Japan \\ 2 Core Research for Evolutional Science and Technology, Japan Science and Technology Agency, Kawaguchi, Japan \\ 3 Genetic Engineering and Functional Genomics Group, Frontier Technology Center, Graduate School of Medicine, Kyoto University, Kyoto, Japan \\ ${ }^{4}$ Department of Psychiatry, Graduate School of Medicine, Kyoto University, Kyoto, Japan \\ 5 Institute for Bioinformatics Research and Development, Japan Science and Technology Agency, Kawaguchi, Japan \\ ${ }^{6}$ Division of Developmental Neurobiology, Department of Physiology and Cell Biology, Kobe University Graduate School of Medicine, Kobe, Japan
}

\section{Edited by:}

Shigeyoshi Itohara, RIKEN Brain

Science Institute, Japan

\section{Reviewed by:}

Alcino J. Silva, University of California, USA

David P. Wolfer, University of Zurich, Switzerland

\section{*Correspondence:}

Tsuyoshi Miyakawa, Division of Systems Medical Science, Institute for Comprehensive Medical Science, Fujita Health University, 1-98

Dengakugakubo, Kutsukake-cho,

Toyoake 470-1192, Japan.

e-mail: miyakawa@fujita-hu.ac.jp

${ }^{\dagger}$ Naoki Matsuo and Nobuyuki Yamasaki have contributed equally to this work.
The alpha-isoform of calcium/calmodulin-dependent protein kinase II ( $\alpha$-CaMKII) is expressed abundantly in the forebrain and is considered to have an essential role in synaptic plasticity and cognitive function. Previously, we reported that mice heterozygous for a null mutation of $\alpha$-CaMKII ( $\alpha$-CaMKII+/-) have profoundly dysregulated behaviors including a severe working memory deficit, which is an endophenotype of schizophrenia and other psychiatric disorders. In addition, we found that almost all the neurons in the dentate gyrus (DG) of the mutant mice failed to mature at molecular, morphological and electrophysiological levels. In the present study, to identify the brain substrates of the working memory deficit in the mutant mice, we examined the expression of the immediate early genes (IEGs), c-Fos and Arc, in the brain after a working memory version of the eight-arm radial maze test. c-Fos expression was abolished almost completely in the DG and was reduced significantly in neurons in the CA1 and CA3 areas of the hippocampus, central amygdala, and medial prefrontal cortex (mPFC). However, c-Fos expression was intact in the entorhinal and visual cortices. Immunohistochemical studies using arc promoter driven dVenus transgenic mice demonstrated that arc gene activation after the working memory task occurred in mature, but not immature neurons in the DG of wild-type mice. These results suggest crucial insights for the neural circuits underlying spatial mnemonic processing during a working memory task and suggest the involvement of $\alpha$-CaMKII in the proper maturation and integration of DG neurons into these circuits.

Keywords: $\alpha$-CaMKII, working memory, dentate gyrus, schizophrenia, immediate-early genes, c-fos

\section{INTRODUCTION}

The alpha isoform of calcium/calmodulin-dependent protein kinase II $(\alpha$-CaMKII) is a serine/threonine protein kinase that is abundant in the forebrain (Lisman et al., 2002). It is activated by $\mathrm{Ca}^{2+} /$ calmodulin, and phosphorylates various protein substrates such as AMPA-type glutamate receptors (AMPARs), synapsin I, tyrosine hydroxylase, L-type $\mathrm{Ca}^{2+}$ channels, and MAP-2. Importantly, $\alpha$-CaMKII can function as a switch molecule with the ability to be autophosphorylated. The phosphorylation of $\alpha$-CaMKII at Thr286 enables the kinase to remain active even after the dissociation of $\mathrm{Ca}^{2+} /$ calmodulin (Griffith, 2004; Irvine et al., 2006). The role of $\alpha$-CaMKII has been examined extensively at both molecular and behavioral levels. Its activity is essential for the induction of long-term potentiation (LTP) in hippocampal slices (Malinow et al., 1989). A number of studies of $\alpha$-CaMKII mutant mice have suggested a function of the kinase in spatial learning and memory (Silva et al., 1992; Bach et al., 1995; Mayford et al., 1996; Giese et al., 1998; Frankland et al., 2001; Elgersma et al., 2002, 2004; Miller et al., 2002; Wang et al., 2003) and in regulating anxiety-like and aggressive behaviors (Chen et al., 1994; Yamasaki et al., 2008; Hasegawa et al., 2009). In addition, we demonstrated that $\alpha$-CaMKII heterozygous knockout ( $\alpha$-CaMKII $+/-)$ mice are severely impaired in working memory (Yamasaki et al., 2008), a trial-specific and short-term memory that can be distinguished from long-lasting reference memory by its transience (Becker et al., 1980). A dysfunction in working memory is a proposed functional endophenotype of schizophrenia and other psychiatric disorders in humans (Goldman-Rakic, 1994). Through our comprehensive behavioral test battery, we also revealed that $\alpha$-CaMKII + /- mice show various dysregulated behaviors including increased locomotor activity, decreased anxiety-like behavior, decreased depressionlike behavior, and periodic mood-change-like behavior (Yamasaki et al., 2008). Biochemical assays revealed several changes such as increased dopamine turnover in the striatum, and increased dopamine D1-like receptor binding in the dentate gyrus (DG) (Yamasaki et al., 2008). In addition, we observed that the molecular, morphological, and electrophysiological features of DG neurons of the $\alpha$-CaMKII+/- mice are strikingly similar to those of immature neurons of wild type mice, and proposed that an "immature DG" in adulthood might serve as a promising candidate endophenotype of schizophrenia and other human psychiatric disorders (Yamasaki et al., 2008). 
The expression of immediate-early genes (IEGs) such as $c$-fos and arc have been used widely as a neuronal activity marker since they are rapidly and transiently induced by neuronal stimuli in the brain (Morgan et al., 1987; Guzowski et al., 2005). Moreover, genetic engineering technology has enabled the visualization of behaviorally activated neurons using reporter genes in mice (Smeyne et al., 1992; Wilson et al., 2002; Barth et al., 2004; Wang et al., 2006; Reijmers et al., 2007; Matsuo et al., 2008). In order to identify the dysfunctional brain regions in $\alpha$-CaMKII $+/-$ mice performing a working memory task, we conducted IEG expression mapping in $\alpha$-CaMKII $+/-$ mice after performance in a working memory version of the eight-arm radial maze test. c-Fos expression was abolished almost completely in the DG and was significantly reduced in neurons in the CA 1 and CA 3 areas of the hippocampus, central amygdala and medial prefrontal cortex (mPFC) compared to the wild-type littermates. Using an arc promoter driven dVenus transgenic mice (Eguchi and Yamaguchi, 2009), we observed that arc gene activation occurred in mature, but not immature neurons in the wild-type DG. These results suggest novel insights for the neural circuits underlying spatial mnemonic processing during a working memory task, and represent further evidence for the involvement of $\alpha$-CaMKII in the proper maturation and integration of DG neurons into these circuits.

\section{MATERIALS AND METHODS ANIMALS}

$\alpha$-CaMKII+/- mice were obtained from Jackson Laboratories (Bar Harbor, Maine). They were bred for more than 20 generations on the C57BL/6J background and kept being backcrossed on the strain continuously thereafter. Mice were housed one per cage after weaning, since the mutants tend to kill their cagemates if grouphoused, in a room with a 12-h light/dark cycle (lights on at 7:00 a.m.) with access to food and water ad libitum. Behavioral testing was performed between 9:00 a.m. and 6:00 p.m. The generation of the Arc-dVenus transgenic mice is detailed in elsewhere (Eguchi and Yamaguchi, 2009). The transgenic mice were obtained by pronuclear microinjection of the transgene into fertilized eggs of BDF1 $(\mathrm{C} 57 \mathrm{BL} / 6 \mathrm{CrSlc} \times \mathrm{DBA} / 2 \mathrm{CrSlc})$. Founder mice were bred for three generations on the BDF1 genetic background and crossed with $\alpha$ CaMKII+/- mice. Behavioral testing procedures were approved by the Animal Care and Use Committee of Kyoto University Graduate School of Medicine.

\section{EIGHT-ARM RADIAL MAZE TEST}

Fully-automated eight-arm radial maze apparatuses (O'Hara \& Co., Tokyo, Japan) were used. The floor of the maze was made of white plastic, and the wall ( $25 \mathrm{~cm}$ high) consisted of transparent plastic. Each arm $(9 \times 40 \mathrm{~cm})$ radiated from an octagonal central starting platform (perimeter $12 \times 8 \mathrm{~cm}$ ) like the spokes of a wheel. Identical food wells ( $1.4 \mathrm{~cm}$ deep and $1.4 \mathrm{~cm}$ in diameter $)$ with pellet sensors were placed at the distal end of each arm. The pellet sensors were able to automatically record pellet intake by the mice. The maze was elevated $75 \mathrm{~cm}$ above the floor and placed in a dimly-lit room with several extra-maze cues. During the experiment, the maze was maintained in a constant orientation. One week before pretraining, animals were deprived of food until their body weight was reduced to $80-85 \%$ of the initial level. Pretraining started on the 8 th day.
Each mouse was placed in the central starting platform and allowed to explore and consume food pellets scattered on the whole maze for a 30-min period (one session per mouse). After completion of the initial pretraining, mice received further pretraining to take a food pellet from each food well after being placed at the distal end of each arm. A trial was finished after the mouse consumed the pellet. This was repeated eight times, using eight different arms, for each mouse. After these pretraining trials, actual maze acquisition trials were performed. In the spatial working memory task of the eightarm radial maze, all eight arms were baited with food pellets. Mice were placed on the central platform and allowed to obtain all eight pellets within $25 \mathrm{~min}$. A trial was terminated immediately after all eight pellets were consumed or 25 min had elapsed. An "arm visit" was defined as traveling more than $5 \mathrm{~cm}$ from the central platform. After each arm choice, all the doors except for the chosen arm were closed. The chosen arm was also closed after the mice returned to the center platform and the mouse was confined in the platform. After 5-s confinement, all the doors are opened. This process was repeated automatically until the termination of a trial. The animals went through one trial per day. For each trial, arm choice, latency to obtain all pellets, distance traveled, number of different arms chosen within the first eight choices, the number of arm revisited, and omission errors were automatically recorded. After the tests, the apparatus were cleaned with diluted sodium hypochlorite solution to prevent a bias due to olfactory cues. Data acquisition, control of guillotine doors, and data analysis were performed by Image RM software. The Image RM was based on the public domain NIH Image program (developed at the U.S. National Institutes of Health and available on the Internet at http://rsb.info.nih.gov/nih-image/), which were modified for each test by Tsuyoshi Miyakawa (available through O’Hara \& Co., Tokyo, Japan).

\section{ANALYSIS OF c-FOS EXPRESSION AFTER THE EIGHT-ARM RADIAL MAZE TEST}

Ten- to thirteen-week-old wild-type mice $(n=14)$ and $\alpha$ CaMKII+/- mice ( $n=14$ ) were subjected to the working memory version of the eight-arm radial maze test. After the mice were trained for 25 trials, the half of these mice were perfusion-fixed with $4 \%$ PFA and $0.5 \%$ picric acid in $0.1 \mathrm{M}$ PBS $90 \mathrm{~min}$ after the end of this task, and the rest group were fixed without any trials in the same day. The brains were removed and further immersion-fixed in the same fixative at $4^{\circ} \mathrm{C}$ overnight and 35 - $\mu \mathrm{m}$-thick coronal sections were prepared on a cryostat (Leica). The immunostaining was performed by incubating free-floating sections with rabbit anti-c-Fos polyclonal antibody (1:20,000 dilution; CALBIOCHEM) at room temperature overnight. Incubation with a biotinylated anti-rabbit IgG made in goat (Vector Laboratories) was followed by incubation with the VECTASTAIN ABC kit (PK-4001, Vector Laboratories) for $1 \mathrm{~h}$ and $0.02 \% \mathrm{DAB} / 0.02 \% \mathrm{H}_{2} \mathrm{O}_{2}$. Sections at each level of +2.10 , $-1.34,-2.30$, and $-2.92 \mathrm{~mm}$ from the bregma were selected for the counting of c-Fos positive cells (see Figure 2Q). Structures of ROI were anatomically defined according to The Mouse Brain in Stereotaxic Coordinates (Franklin and Paxinos, 2008). Images of specified regions or subdivisions were acquired using a light microscope (Zeiss Axioplan2) outfitted with a CCD camera bilaterally by an experimenter blind to the genotypes and behavioral condition. Thresholded images of c-Fos immunopositive nuclei 
were automatically counted using ImageJ. (http://rsb.info.nih. gov/ij/).

\section{ANALYSIS OF Arc EXPRESSION AFTER THE EIGHT-ARM RADIAL MAZE TEST}

To generate experimental mice, $\alpha$-CaMKII+/- mice were crossed with Arc-dVenus transgenic mice (Eguchi and Yamaguchi, 2009), and Arc-dVenus positive and $\alpha$-CaMKII+/- mice were compared with Arc-dVenus positive and $\alpha$-CaMKII $+/+$ mice. The working memory version of the eight-arm radial maze test was performed with 10 -week-old male mice. After the mice were trained for 37 trials, these mice were perfusion-fixed with $4 \%$ PFA and $0.5 \%$ picric acid in $0.1 \mathrm{M}$ PBS $5 \mathrm{~h}$ after the end of this task. The brains were removed and 35- $\mu$ m-thick coronal sections were prepared on a cryostat (Leica). Sections were immunostained for calbindin and calretinin as described above, and observed using a confocal laser-scanning microscope (LSM5, Zeiss).

\section{STATISTICAL ANALYSIS}

Statistical analysis was conducted using StatView (SAS Institute, Cary, NC). Data were analyzed by ANOVA unless noted otherwise. Values in tables and graphs were expressed as mean \pm SEM.

\section{RESULTS}

REDUCED C-FOS EXPRESSION AFTER THE WORKING MEMORY VERSION OF THE RADIAL MAZE TASK IN $\alpha$-CAMKII+/-MICE

In a previous study, we reported that $\alpha$-CaMKII+/- mice show a profound deficit in spatial working memory. In the working memory version of the eight-arm radial maze task, wild-type mice improved their performance by training in the number of different arm choices in the first eight entries and made significantly fewer revisiting errors than the mutant mice (Yamasaki et al., 2008). To gain insight into the brain regions impaired in the mutant mice, we performed c-Fos mapping in wild-type littermates and $\alpha$-CaMKII $+/-$ mice. The expression of immediate-early genes (IEGs) such as $c$-fos and arc have been used widely as neuronal activity markers since they are rapidly and transiently induced by neuronal stimuli in the brain (Morgan et al., 1987; Guzowski et al., 2005). Mice were subjected to a working memory version of the eight-arm radial maze test (Figure 1). At 90 min after the mice were trained for 25 trials, we performed immunohistochemistry for c-Fos (Figure 2). In wild-type mice, the density of c-Fospositive cells was increased in most regions examined, including the CA1, CA3, and DG regions of the hippocampus, entorhinal cortex, visual cortex, central and medial amygdaloid nucleus, and

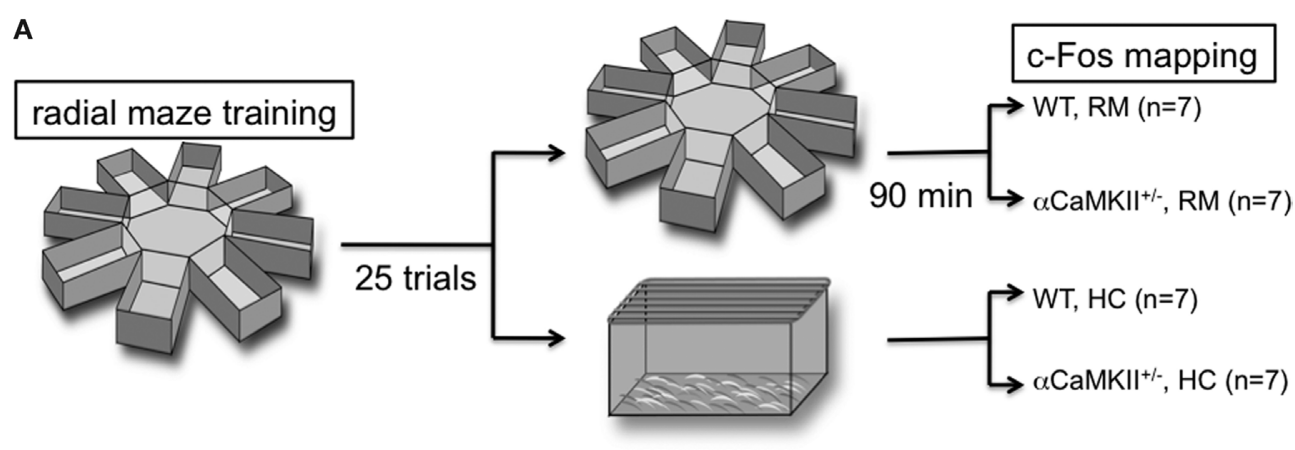

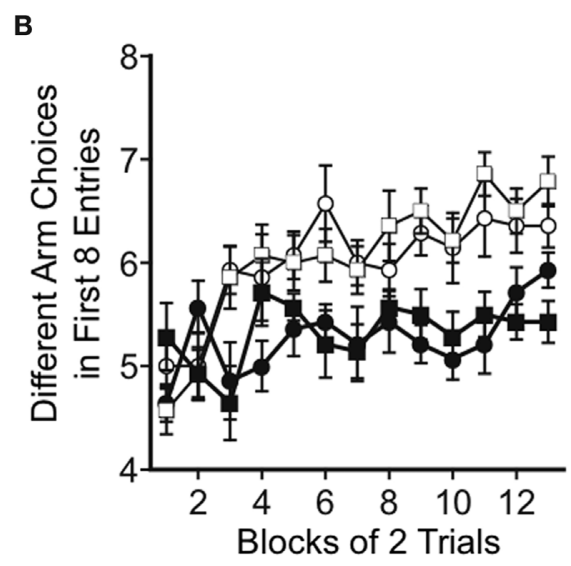

FIGURE 1 |Working memory version of the eight-arm radial maze test. (A) Experimental design. Wild-type $(n=14)$ and $\alpha$-CaMKII+/- mice $(n=14)$ were subjected to the working memory version of the eight-arm radial maze test for 25 trials. Ninety minutes after the last trial, half of the mice were sacrificed for c-Fos immunostaining $(\mathrm{RM})$, and the rest group were sacrificed directly from their home cage without any trials in the same day $(\mathrm{HC})$. (B,C) Both wild-type and the mutant mice improved their performance by training in the number of different

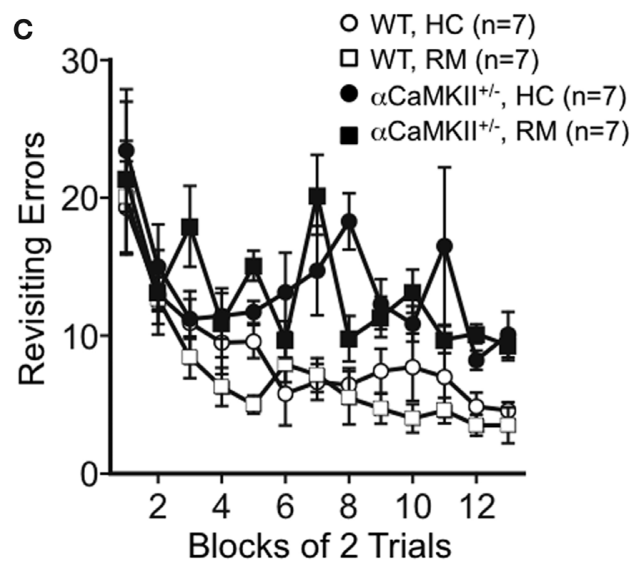

arm choices in the first eight entries [WT: $F(12,156)=14.069, P<0.0001$, $\alpha$-CaMKII+/-: $F(12,156)=1.830, P=0.0477$, repeated measures ANOVA]. However, the $\alpha$-CaMKII+/- mice performed significantly worse than control wild type littermates with respect to the number of different arm choices in the first eight entries $[F(1,26)=29.825, P<0.0001$, repeated measures ANOVA $]$ and made significantly more revisiting errors $[F(1,26)=38.896, P<0.0001$, repeated measures ANOVA] than control mice. Error bars indicate SEM. 

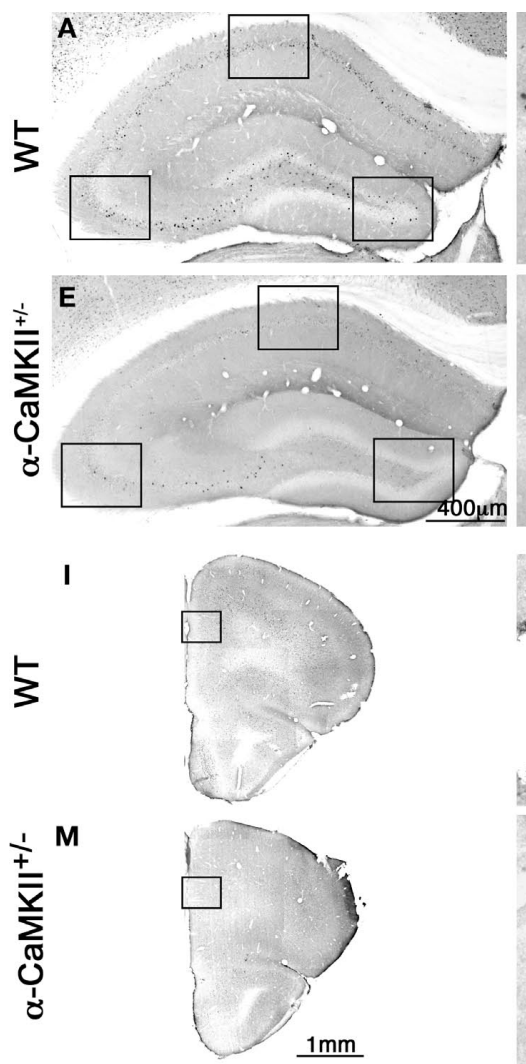

Q
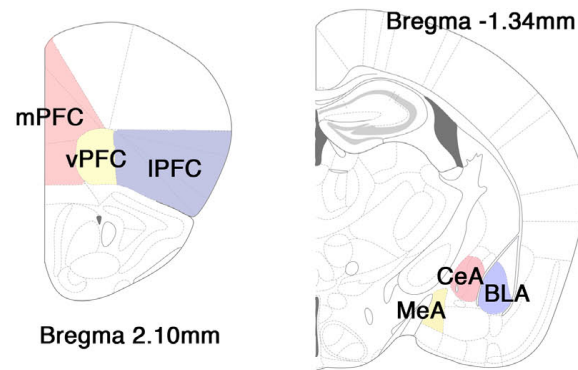

FIGURE 2 | Photomicrographs of c-Fos expression after the eight-arm radial maze test. Representative photomicrographs of c-Fos expression after the working memory task in the hippocampus (A), dentate gyrus (B), CA1 area (C), CA3 area (D), frontal cortex (I), and mPFC (J), entorhinal cortex (K), and basolateral amygdala (L) in the wild-type mice. Photomicrographs of c-Fos expression after the working memory task in the hippocampus (E), dentate gyrus (F), CA1 area (G), CA3 area (H), frontal cortex (M), and mPFC (N), entorhinal cortex (O), and basolateral amygdala (P) in the $\alpha$-CaMKII+/- mice. (Q) Schematic drawings of mouse brain coronal sections adapted from
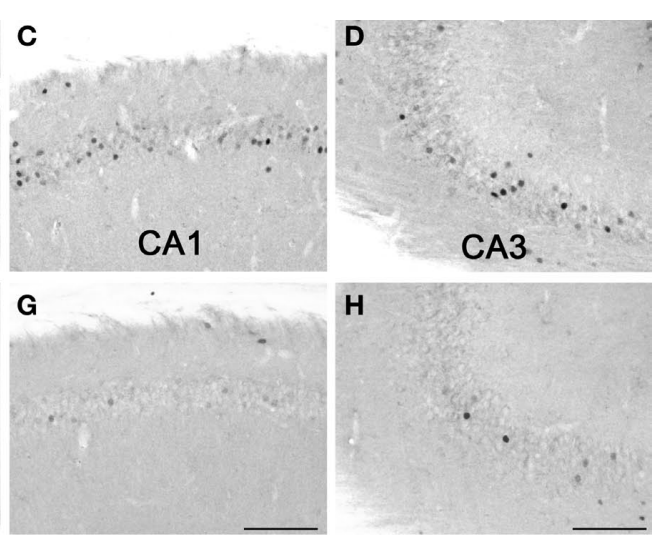

$\underline{100 \mu \mathrm{m}}$
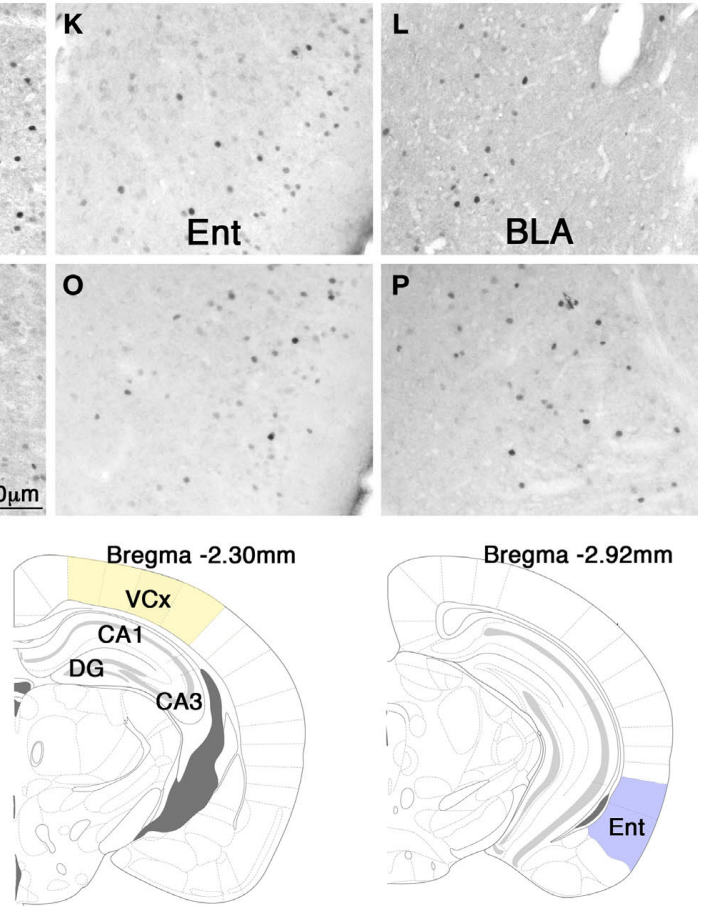

(Franklin and Paxinos, 2008) showing the regions of interest selected for measurement of c-Fos positive cells. mPFC, medial prefrontal cortex (cingulate cortex, prelimbic cortex, medial orbital cortex); vPFC, ventral prefrontal cortex (ventral orbital cortex); IPFC, lateral prefrontal cortex (lateral orbital cortex, frontal cortex area3, agranular insular cortex dorsal and ventral part); CeA, central amygdaloid nucleus; MeA, medial amygdaloid nucleus; BLA, basolateral amygdaloid nucleus; VCx, visual cortex (primary and secondary visual cortex); Ent, entorhinal cortex (dorsolateral and dorsintermed entorhinal cortex). medial and lateral prefrontal cortex compared with home cage control animals (Figure 3A). Although elevated c-Fos expression was observed in the entorhinal cortex, visual cortex, basolateral amygdaloid nucleus, and medial and ventral prefrontal cortex in the $\alpha$-CaMKII $+/-$ mice after the working memory task (Figure 3B), a significant increase was not observed in CA1 (genotype effect, $F(1,12)=10.941, P=0.0062)$, CA3 (genotype $\times$ task interaction, $F(1,24)=7.304, P=0.0124$, genotype effect, $F(3,24)=12.103$, $P<0.0001$ ), DG (genotype $\times$ task interaction, $F(1,24)=17.332$, $P=0.0003$, genotype effect, $F(3,24)=37.812, P<0.0001)$, central amygdala $($ genotype $\times$ task interaction, $F(1,24)=6.370, P=0.0186$, genotype effect, $F(3,24)=7.230, P=0.0068)$, and medial prefrontal cortex (genotype effect, $F(1,12)=7.601, P=0.0174$ ) compared to wild-type mice (Figure 3C). These results suggest that the neural activation in these regions activated by spatial working memory task is impaired in the mutant mice. The absence of a pronounced increase in c-Fos expression in some brain regions in the mutant mice is likely due to the silenced activities of neuronal circuits rather than a disrupted molecular machinery of c-Fos expression in the $\alpha$-CaMKII $+/-$ mice, because the expression levels of c-Fos in 


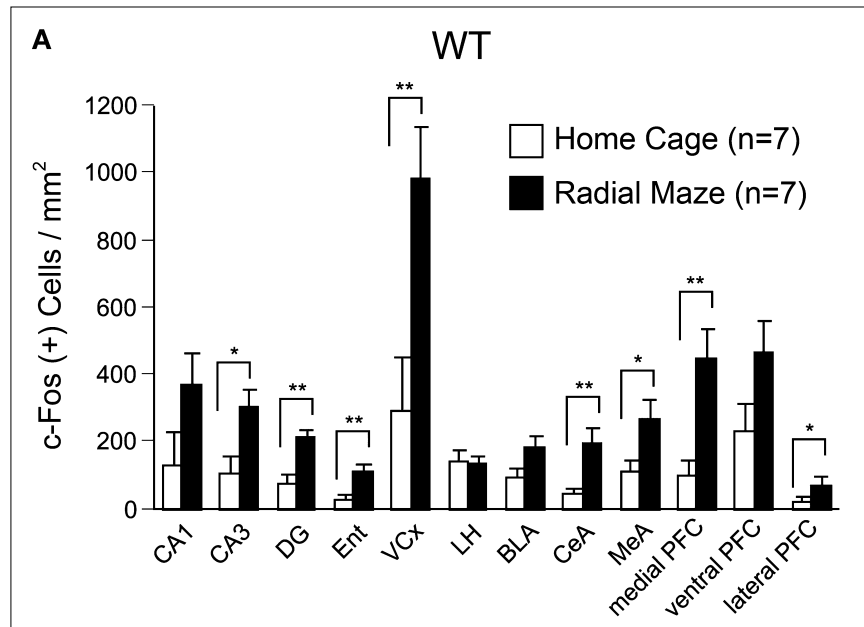

C

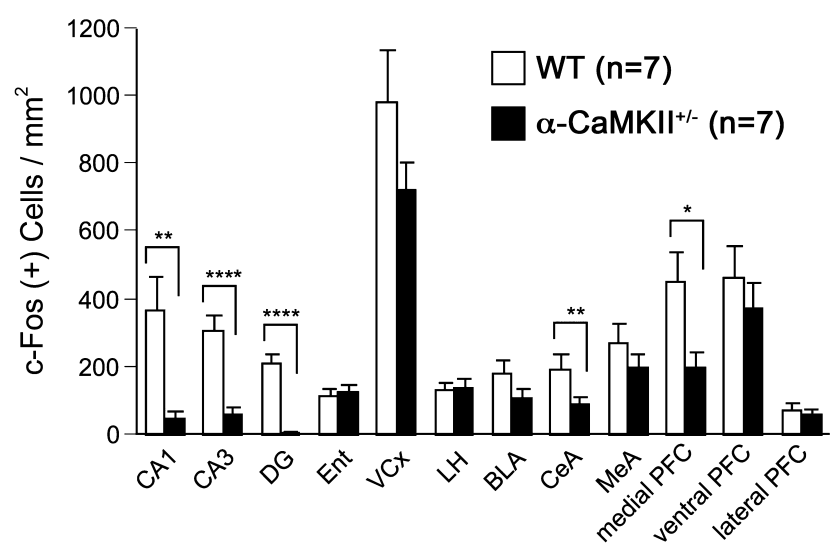

FIGURE 3 | Densities of c-Fos stained cells after the eight-arm radial maze test. (A) Densities of c-Fos immunoreacive cells in wild-type mice. (B) Densities of c-Fos immunoreactive cells in the $\alpha$-CaMKII+/- mice. (C) Densities of c-Fos immunoreacive cells after the working memory task. (D) Densities of c-Fos immunoreacive cells in the home cage control animals. DG, dentate

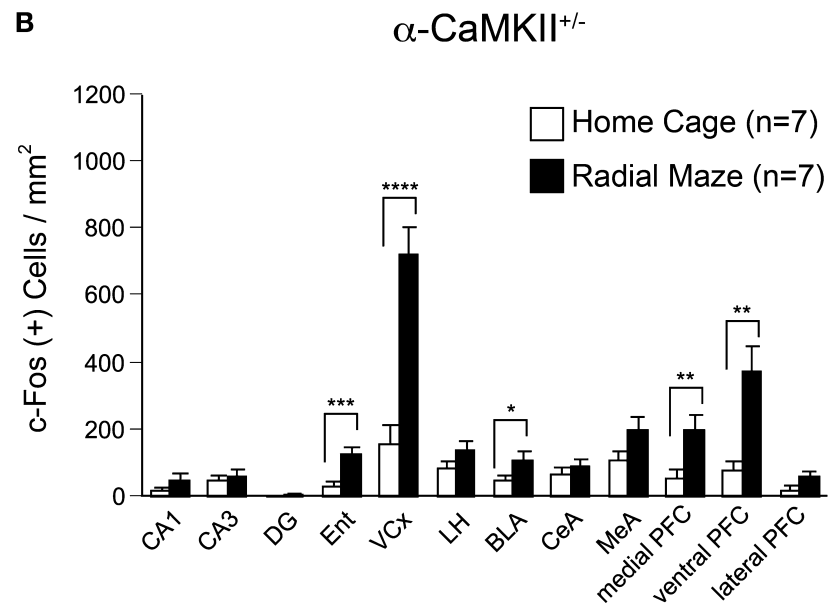

D

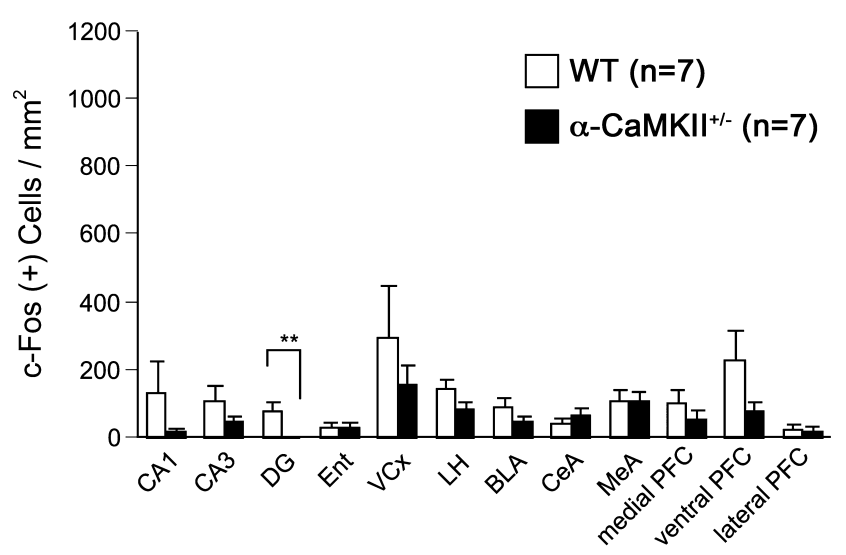

gyrus; Ent, entorhinal cortex; VCx, visual cortex (primary and secondary visual cortex); LH, lateral hypothalamus; BLA, basolateral amygdaloid nucleus; CeA, central amygdaloid nucleus; MeA, medial amygdaloid nucleus; PFC, prefrontal cortex. ${ }^{*} P<0.05,{ }^{*} P<0.01,{ }^{* *} P<0.001,{ }^{* * *} P<0.0001$. Error bars indicate SEM. the home cage control animals was comparable between the wildtype and the mutant mice in the brain except for the DG, where c-Fos expression was almost completely abolished (Figure 3D). In addition, previous reports showed no significant difference in the density of c-Fos positive neurons after acquisition of contextual fear conditioning in the CA1, CA3, cortex, and amygdala between wild-type and $\alpha$-CaMKII+/- mice (Frankland et al., 2004).

\section{ArC-VENUS EXPRESSION WAS DETECTED IN MATURE NEURONS, BUT NOT IMMATURE NEURONS IN THE DG AFTER WORKING MEMORY TASK}

Recent genetic engineering technology has enabled the visualization of behaviorally activated neurons using reporter genes in mice (Smeyne et al., 1992; Wilson et al., 2002; Barth et al., 2004; Wang et al., 2006; Reijmers et al., 2007; Matsuo et al., 2008). Here, we used Arc-dVenus transgenic mice that expressed destabilized Venus fluorescent protein driven by the arc promoter to detect in vivo neuronal responsiveness to behavioral tasks (Eguchi and Yamaguchi, 2009). We investigated dVenus expression in the Arc-dVenus+/
$\alpha$-CaMKII+/- mice, obtained by crossing the $\alpha$-CaMKII $+/-$ mice with the Arc-dVenus transgenic mice, after performing the working memory task. In this experiment, we focused on the DG since the c-Fos mapping studies demonstrated the most striking changes in the DG. Similar to the c-Fos immunohistochemistry results, Arc-dVenus-positive cells were abolished completely in the DG of Arc-dVenus+/ $\alpha$-CaMKII+/- home cage control mice and after the working memory task (Figures 4 and 5A,B). In the DG of the ArcdVenus $+/ \alpha$-CaMKII $+/+$ mice, the density of dVenus-positive cells was significantly increased after the working memory task compared to that of mice that remained in their home cage (Figure 4: task effect, $F(1,6)=7.086, P=0.0374)$. These results indicate that dVenus is induced in the DG in response to behavioral activity in the Arc-dVenus transgenic mice.

In the DG of Arc-dVenus+/ $\alpha$-CaMKII+/+ mice, dVenus-positive cells were observed in the granule cell layer (GCL), but not in the subgranular zone (SGZ) (Figures 5C-L). In the adult DG, new neurons are continuously generated in the SGZ and migrate into 


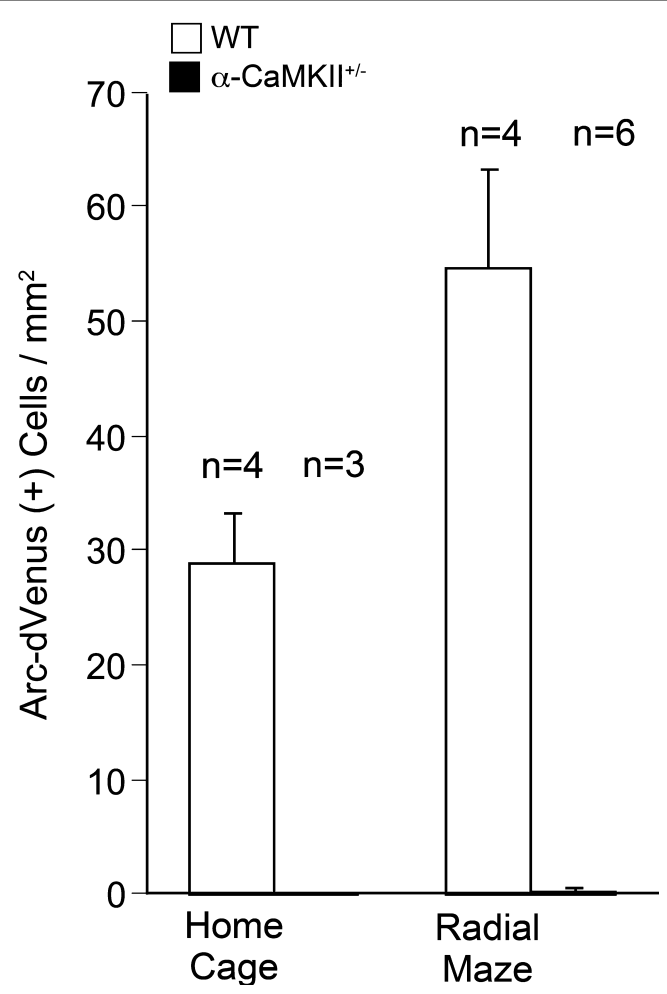

FIGURE 4 | Densities of Arc-dVenus positive cells in the DG after the eight-arm radial maze test. Few cells in the DG of $\alpha$-CaMKII+/- mice showed Arc-dVenus fluorescence either in the home cage control animals or after the working memory task. Error bars indicate SEM.

the GCL during maturation (Ming and Song, 2005). To further characterize the stage of differentiation of the dVenus positive cells in the DG after the working memory task, we performed immunohistochemistry using antibodies against calretinin and calbindin, which are molecular markers for immature and mature neurons, respectively (Kuhn et al., 1996; Brandt et al., 2003; Kempermann et al., 2004). The co-labeling study revealed that the dVenus positive cells were localized separately from calretinin-positive cells (Figures 5C,E,G-I), but were colocalized with calbindin (Figures 5D,F,J-L). These results indicate that the working memory task-induced dVenus expression occurs in mature, but not immature neurons in the DG.

\section{DISCUSSION}

Consistent with previous reports (Vann et al., 2000; Touzani et al., 2003), our results show that c-Fos expression is increased in the visual cortex, entorhinal cortex, hippocampus, central amygdala, and mPFC after the working memory task in wildtype mice. However, in $\alpha$-CaMKII $+/-$ mice, increases of $c$-Fos expression were not observed in the DG, CA1, and CA3 regions of the hippocampus, $\mathrm{mPFC}$, and central amygdala. These results suggest that these brain regions in the mutant mice have functional activation deficits in response to the working memory task, and that these regions are good candidates for the brain regions responsible for the working memory deficits in the mutant mice.

\section{SUPPRESSED c-FOS INDUCTION IN THE HIPPOCAMPUS OF} $\alpha$ CAMKII+/-MICE

The hippocampus is a critical structure involved in spatial working/ episodic-like memory in both rodents and primates (Becker et al., 1980; Olton et al., 1982; Goldman-Rakic, 1995). The hippocampus contains parallel excitatory pathways referred to as trisynaptic pathway and the monosynaptic pathway. In the trisynaptic pathway, information flows from the superficial layer of the entorhinal cortex to the DG then to CA3, and finally to CA1. In the monosynaptic pathway, CA1 neurons receive inputs directly from the entorhinal cortex layer III. In the hippocampus of $\alpha$-CaMKII+/mice, increased c-Fos expression was compromised in the DG, as well as CA1 and CA3 neurons after the working memory task. However, in the entorhinal cortex of $\alpha$-CaMKII $+/-$ mice, $c$-Fos expression was induced significantly to the same extent as that observed in the wild-type mice after the working memory task. These results indicate at least two possibilities for the suppressed c-Fos induction in the mutant hippocampus. First, the DG may be impaired, while the CA 1 and CA 3 regions are intact. The second possibility is that neuronal functions in the whole hippocampus including the CA1, CA3 areas, and the DG are disturbed. We consider the first possibility to be more likely because c-Fos expression is induced in CA1 and CA 3 in $\alpha$-CaMKII $+/-$ mice after acquisition of contextual fear conditioning or electric foot shock to the same extent as that in the wild-type mice (Frankland et al., 2004; Yamasaki et al., 2008). These results suggest that the CA1 and CA3 neurons of the mutant mice have an intact ability to express c-Fos in response to behavioral stimuli. In agreement with our c-Fos expression data, electrophysiological studies have shown that Schaffer collateral stimulation (CA3 to CA1 pathway) produced stable LTP in the CA1 region of the $\alpha$-CaMKII+/- mice (Silva et al., 1996; Frankland et al., 2001), whereas DG granule cell excitability and mossy fiber (MF) synaptic transmission was abnormal in the mutant mice (Yamasaki et al., 2008). In addition, the expression of c-Fos and the arc promoter driven dVenus transgene were abolished almost completely in the mutant DG, even in the home cage control mice. Taken together, these results suggest that dysfunction of the DG is the primary cause for the suppressed c-Fos induction within the mutant hippocampus after the working memory task.

Increased c-Fos expression in the entorhinal cortex and suppressed expression in the CA1 region of the $\alpha$-CaMKII+/- mice after the working memory task suggest that activation of the monosynaptic pathway is not sufficient to activate CA1 neurons during the working memory task, and that activation of the trisynaptic pathway is required. Considering a severe impairment of $\alpha$-CaMKII+/- mice in working memory, these results suggest that the trisynaptic pathway plays a more important role in performing the working memory task than the monosynaptic pathway. This idea is consistent with previous studies. The size of the hippocampal intra- and infrapyramidal MF terminal fields correlates with performance in the spatial working memory task of the radial maze (Crusio et al., 1987; Schwegler et al., 1990). A lesion of the DG results in deficits in working memory using the radial maze test (Walsh et al., 1986; McLamb et al., 1988; Emerich and Walsh, 1989). Mice with an NMDAR deletion restricted to CA3 pyramidal cells are impaired in rapid memory acquisition and storage of a 

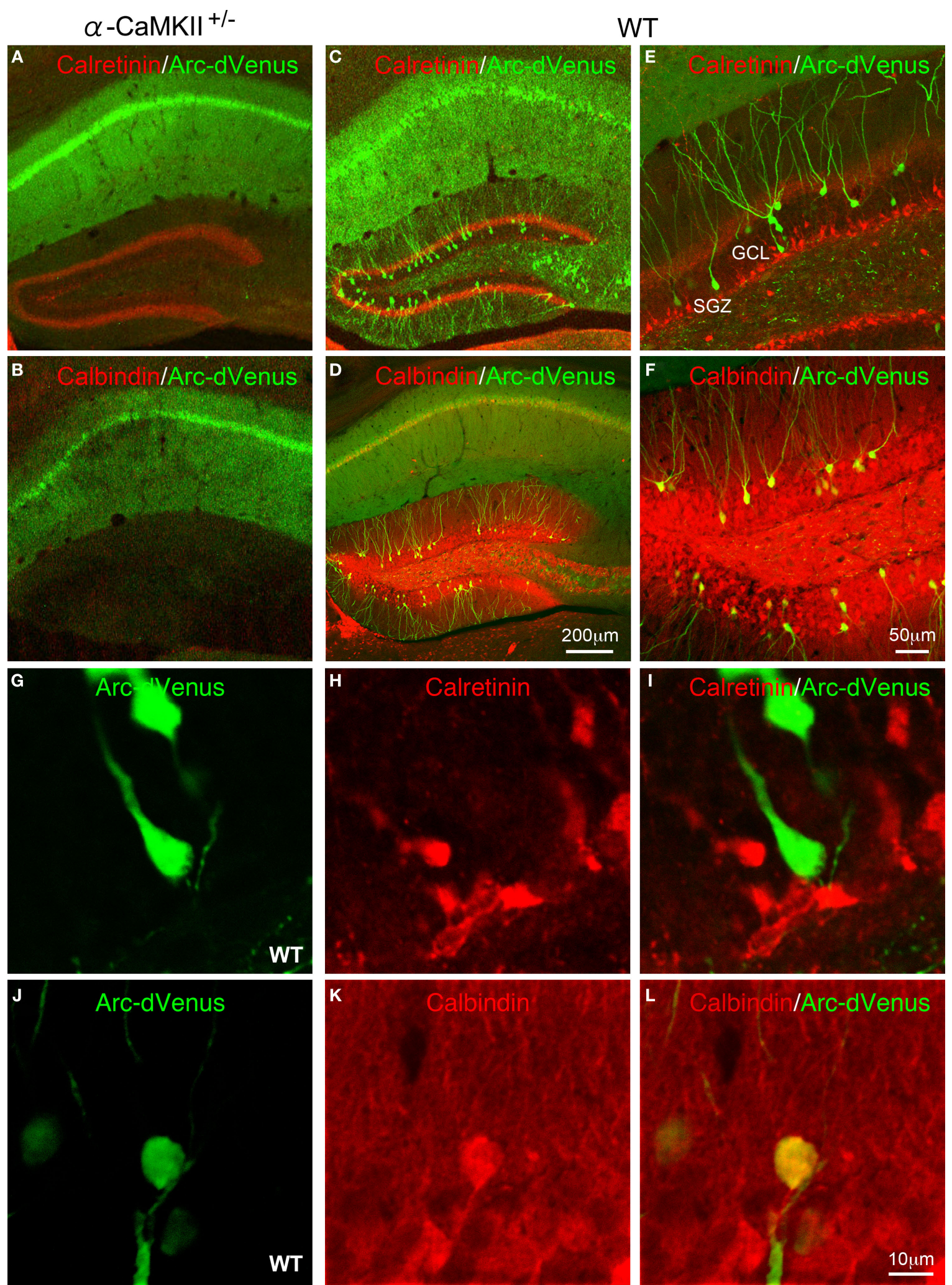

FIGURE 5 | Confocal images of Arc-dVenus and neuronal differentiation marker expression after the eight-arm radial maze test. Expression of Arc-dVenus (green) and calretinin (A) or calbindin (B) in the hippocampus of
$\alpha$-CaMKII+/- mice. Expression of Arc-dVenus (green) and calretinin (C) or calbindin (D) in the hippocampus of wild-type mice. Expression of Arc-dVenus (green) and calretinin $(\mathbf{E}, \mathbf{G}-\mathbf{I})$ or calbindin $(\mathbf{F}, \mathbf{J}-\mathbf{L})$ in the DG of wild-type mice. 
one-time experience in a delayed matching-to-place version of the Morris water maze task (Nakazawa et al., 2003).

\section{MATURITY OF DG NEURONS AND WORKING MEMORY PERFORMANCE}

In the mutant hippocampus, MF-CA3 synapses were poorly developed at the ultrastructural level, and abnormal transmission including an increased basal transmission and a greatly reduced MF facilitation was observed at MF-CA3 synapses (Yamasaki et al., 2008). At the molecular level, we demonstrated that immunopositive cells for polysialic acid-NCAM (PSA-NCAM), a marker for late-stage progenitors and immature neurons, and calretinin, a marker for immature neurons, were significantly increased in the mutant DG, while the density of calbindin-positive cells, a marker for mature neurons in the DG, was dramatically reduced (Yamasaki et al., 2008). In wild-type mice, using co-labeling of arc promoter-induced dVenus and neuronal differentiation markers, our results showed that DG granule cells that were active during the working memory task were immunoreactive for calbindin, but not for calretinin whose expression is barely detected (only 6\%) in 4 -week-old neurons (Brandt et al., 2003). This finding is consistent with previous reports showing that adult-born neurons are functionally integrated into the neuronal circuits after a maturation period of approximately 4 weeks (Carlen et al., 2002; Jessberger and Kempermann, 2003; Kee et al., 2007). These findings indicate that young immature neurons do not participate in hippocampusdependent cognitive behaviors. Thus, the dysfunction of the DG in $\alpha$-CaMKII $+/-$ mice is likely to result from immaturity of the DG neurons. In support of this hypothesis, a recent study has proposed an association between the aging-related working memory impairment and the retarded maturation of newly born DG neurons in aged rats (Nyffeler et al., 2008).

\section{SUPPRESSED c-FOS INDUCTION IN THE mPFC OF $\alpha$-CAMKII+/-MICE}

Outside the hippocampus, working memory task-induced c-Fos expression was compromised in the MPFC and central amygdala in the mutant mice. The mPFC has been suggested to play a key role in working memory (Goldman-Rakic, 1995), whereas the amygdala has not been shown to participate (Becker et al., 1980; Friedman and Goldman-Rakic, 1988; Bianchin et al., 1999). Anatomically, there is a monosynaptic pathway exists between the hippocampus and the mPFC that originates from the hippocampal CA1/ subiculum fields and innervates the prelimbic/medial orbital areas of the PFC (Thierry et al., 2000). In addition, evidence suggests critical functional interactions between these regions in working memory. For example, disruption of the hippocampal-prefrontal pathway impairs performance on a spatial working memory task in rats (Floresco et al., 1997). Additionally, simultaneous tetrode recordings from the rat hippocampus and MPFC revealed that the activity in these different regions may be synchronized during a spatial working memory task (Jones and Wilson, 2005). In a previous study, c-Fos and Zif268 induction in the mPFC of $\alpha$ CaMKII+/- mice after acquisition of contextual fear conditioning was normal (Frankland et al., 2004). This suggests that neurons in the mutant $\mathrm{mPFC}$ are functional though we need to be careful about the difference of genetic background between our mice (more than 20 generations on the C57BL/6J background) and those of Frankland's group (50\% C57Bl/6NTacfBr and 50\% 129Sv/J). In the fear conditioning training, it is possible that the mPFC neurons were activated through the thalamus-PFC pathway (Oyoshi et al., 1996). The reduced c-Fos expression in the MPFC after the working memory task in the $\alpha$-CaMKII+/- mice is likely to be due to deficits in hippocampal networks resulting from the DG functional impairment. Alternatively, DG dysfunction during development may have caused secondary functional alterations in other interconnected areas including the mPFC (Lipska and Weinberger, 1993). Dysfunction of these functional neuronal networks among the hippocampus, $\mathrm{mPFC}$, and other regions could have resulted in the working memory deficits in the $\alpha$-CaMKII+/- mice.

\section{IMPLICATIONS FOR NEUROPSYCHIATRIC DISORDERS}

Working memory has been suggested to be compromised in multiple psychiatric disorders and may contribute to the behavioral and cognitive deficits associated with the disorders (Goldman-Rakic, 1994). Abnormalities in the hippocampus, mPFC, and amygdala, where c-Fos induction was suppressed in the $\alpha$-CaMKII $+/-$ mice during working memory performance, has been demonstrated in patients with psychiatric disorders (Torrey and Peterson, 1974; Weinberger, 1988). Schizophrenia, bipolar disorder, and other related psychiatric disorders are considered to be biologically heterogeneous populations, due to the limitation of the current methods of psychiatric diagnostic methods. Thus, an endophenotype-based analysis would be preferable for establishing biological characteristics for the classification of psychiatric disorders, rather than an analysis based on current diagnostic methods (Gottesman and Gould, 2003). Establishing animal models of psychiatric disorders would be very useful to identify endophenotypes and mechanisms associated with the disorders. The $\alpha$-CaMKII + /- mice may provide an animal model of schizophrenia with face and construct validity. These mice fulfill many of the criteria for face validity, such as multiple abnormal behaviors including a severe working memory deficits and hyperactivity. In terms of the construct validity, an increased dopamine turnover and increased dopamine D1like receptor binding in the $\alpha$-CaMKII + - - mice are consistent with the dopamine hypothesis (Carlsson et al., 2001). A critical role of $\alpha$-CaMKII in glutamate receptor signaling pathways and synaptic plasticity (Lisman et al., 2002) is consistent with the glutamate hypothesis (Goff and Coyle, 2001). Moreover, the DG immaturity agrees with the neurodevelopmental hypothesis (Weinberger, 1996). Our findings that neuronal activities in the hippocampus, mPFC, and central amygdala in $\alpha$-CaMKII+/- mice were suppressed during the working memory task increases the face validity of the mice as an animal model for schizophrenia.

\section{ACKNOWLEDGEMENTS}

This work was supported by KAKENHI (Grant-in-Aid for Scientific Research) on Priority Areas "Systems Genomics" (20016013), "Pathomechanisms of Brain Disorders" (20023017), Young Scientists A (16680015), Exploratory Research (19653081), and Integrative Brain Research (IBR-shien) from the Ministry of Education, Culture, Sports, Science and Technology (MEXT) of Japan, Promotion of Fundamental Studies in Health Sciences of the National Institute of Biomedical Innovation (NIBIO), Neuroinformatics Japan Center (NIJC), and by grants from CREST \& BIRD of Japan Science and Technology Agency (JST). 


\section{REFERENCES}

Bach, M. E., Hawkins, R. D., Osman, M., Kandel,E. R., and Mayford,M. (1995). Impairment of spatial but not contextual memory in CaMKII mutant mice with a selective loss of hippocampal LTP in the range of the theta frequency. Cell 81, 905-915.

Barth, A. L., Gerkin, R. C., and Dean, K. L. (2004). Alteration of neuronal firing properties after in vivo experience in a FosGFP transgenic mouse. J. Neurosci. 24, 6466-6475.

Becker, J. T., Walker, J. A., and Olton, D. S. (1980). Neuroanatomical bases of spatial memory. Brain Res. 200, 307-320

Bianchin, M., Mello e Souza, T., Medina, J. H., and Izquierdo, I. (1999). The amygdala is involved in the modulation of long-term memory, but not in working or short-term memory. Neurobiol. Learn. Mem. 71, 127-131.

Brandt, M. D., Jessberger, S., Steiner, B., Kronenberg, G., Reuter, K., BickSander, A., von der Behrens, W., and Kempermann, G. (2003). Transient calretinin expression defines early postmitotic step of neuronal differentiation in adult hippocampal neurogenesis of mice. Mol. Cell. Neurosci. 24, 603-613.

Carlen, M., Cassidy, R. M., Brismar, H., Smith, G. A., Enquist, L. W., and Frisen, J. (2002). Functional integration of adult-born neurons. Curr. Biol. 12, 606-608.

Carlsson, A., Waters, N., HolmWaters, S., Tedroff, J., Nilsson, M., and Carlsson, M. L. (2001). Interactions between monoamines, glutamate, and GABA in schizophrenia: new evidence. Annu. Rev. Pharmacol. Toxicol. 41, 237-260.

Chen, C., Rainnie, D. G., Greene, R. W., and Tonegawa, S. (1994). Abnormal fear response and aggressive behavior in mutant mice deficient for alphacalcium-calmodulin kinase II. Science 266, 291-294.

Crusio, W.E.,Schwegler,H., and Lipp, H. P. (1987). Radial-maze performance and structural variation of the hippocampus in mice: a correlation with mossy fibre distribution. Brain Res. 425, 182-185.

Eguchi, M., and Yamaguchi, S. (2009). In vivo and in vitro visualization of gene expression dynamics over extensive areas of the brain. Neuroimage 44, 1274-1283.

Elgersma, Y., Fedorov, N. B., Ikonen, S., Choi, E. S., Elgersma, M. Carvalho, O. M., Giese, K. P., and Silva, A. J. (2002). Inhibitory autophosphorylation of CaMKII controls PSD association, plasticity, and learning. Neuron 36, 493-505.
Elgersma, Y., Sweatt, J. D., and Giese, K. P. (2004). Mouse genetic approaches to investigating calcium/calmodulindependent protein kinase II function in plasticity and cognition. J. Neurosci. 24, 8410-8415.

Emerich, D. F., and Walsh, T. J. (1989). Selective working memory impairments following intradentate injection of colchicine: attenuation of the behavioral but not the neuropathological effects by gangliosides GM1 and AGF2. Physiol. Behav. 45, 93-101.

Floresco, S. B., Seamans, J. K., and Phillips, A. G. (1997). Selective roles for hippocampal, prefrontal cortical, and ventral striatal circuits in radialarm maze tasks with or without a delay. J. Neurosci. 17, 1880-1890.

Frankland, P. W., Bontempi, B., Talton, L. E., Kaczmarek, L., and Silva, A. J. (2004). The involvement of the anterior cingulate cortex in remote contextual fear memory. Science 304, 881-883.

Frankland, P. W., O’Brien, C., Ohno, M., Kirkwood, A., and Silva, A. J. (2001). Alpha-CaMKII-dependent plasticity in the cortex is required for permanent memory. Nature 411, 309-313.

Franklin, K. B.J., and Paxinos, G. (2008). The Mouse Brain in Stereotaxic Coordinates. San Diego, Academic Press.

Friedman,H.R., and Goldman-Rakic, P. S. (1988). Activation of the hippocampus and dentate gyrus by workingmemory: a 2-deoxyglucose study of behaving rhesus monkeys. J. Neurosci. 8, 4693-4706.

Giese, K. P., Fedorov, N. B., Filipkowski, R. K., and Silva,A.J. (1998). Autophosphorylation at Thr286 of the alpha calcium-calmodulin kinase II in LTP and learning. Science 279, 870-873.

Goff, D. C., and Coyle, J. T. (2001). The emerging role of glutamate in the pathophysiology and treatment of schizophrenia. Am. J. Psychiatry 158, 1367-1377.

Goldman-Rakic, P. S. (1994). Working memory dysfunction in schizophrenia. J. Neuropsychiatry Clin. Neurosci. 6, 348-357.

Goldman-Rakic, P. S. (1995). Cellular basis of working memory. Neuron 14 , 477-485.

Gottesman, I. I., and Gould, T. D. (2003) The endophenotype concept in psychiatry: etymology and strategic intentions. Am. J. Psychiatry 160, 636-645.

Griffith, L. C. (2004). Regulation of calcium/calmodulin-dependent protein kinase II activation by intramolecular and intermolecularinteractions. J. Neurosci. 24, 8394-8398.

Guzowski, J. F., Timlin, J. A., Roysam, B. McNaughton, B. L., Worley, P. F. and Barnes, C. A. (2005). Mapping behaviorally relevant neural circuits with immediate-early gene expression. Curr. Opin. Neurobiol. 15, 599-606.

Hasegawa, S., Furuichi, T., Yoshida, T., Endoh, K., Kato, K., Sado, M. Maeda, R., Kitamoto, A., Miyao, T. Suzuki, R., Homma, S., Masushige, S. Kajii, Y., and Kida, S. (2009). Transgenic up-regulation of alpha-CaMKII in forebrain leads to increased anxietylike behaviors and aggression. Mol Brain 2, 6 .

Irvine, E. E., von Hertzen, L. S., Plattner, F. and Giese, K. P. (2006). alphaCaMKII autophosphorylation: a fast track to memory. Trends Neurosci. 29 459-465.

Jessberger, S., and Kempermann, G (2003).Adult-born hippocampal neurons mature into activity-dependent responsiveness. Eur. J. Neurosci. 18, 2707-2712.

Jones, M. W., and Wilson, M. A (2005). Theta rhythms coordinate hippocampal-prefrontal interactions in a spatial memory task. PLoS Biol. 3, e402. doi: 10.1371/journal. pbio.0030402.

Kee, N., Teixeira, C. M., Wang, A. H., and Frankland, P. W. (2007). Preferentia incorporation of adult-generated granule cells into spatial memory networks in the dentate gyrus. Nat Neurosci. 10, 355-362.

Kempermann, G., Jessberger, S., Steiner, B. and Kronenberg, G. (2004). Milestones of neuronal development in the adult hippocampus. Trends Neurosci. 27, 447-452.

Kuhn, H. G., Dickinson-Anson, H., and Gage, F. H. (1996). Neurogenesis in the dentate gyrus of the adult rat: age-related decrease of neuronal progenitor proliferation. J. Neurosci. 16 2027-2033.

Lipska, B. K., and Weinberger, D. R. (1993) Delayed effects of neonatal hippocampal damage on haloperidol-induced catalepsy and apomorphine-induced stereotypic behaviors in the rat. Brain Res. Dev. Brain Res. 75, 213-222.

Lisman, J., Schulman, H., and Cline, $\mathrm{H}$ (2002). The molecular basis of CaMKII function in synaptic and behavioural memory. Nat. Rev. Neurosci. 3, 175-190.

Malinow, R., Schulman, H., and Tsien, R. W. (1989). Inhibition of postsynaptic PKC or CaMKII blocks induction but not expression of LTP. Science 245, 862-866.

Matsuo, N., Reijmers, L., and Mayford, M. (2008).Spine-type-specific recruitment of newly synthesized AMPA receptors with learning. Science 319, 1104-1107.

Mayford, M., Bach, M. E., Huang, Y. Y. Wang, L., Hawkins, R. D., and Kandel, E. R. (1996). Control of memory formation through regulated expression of a CaMKII transgene. Science 274, 1678-1683.

McLamb, R. L., Mundy, W. R., and Tilson, H. A. (1988). Intradentate colchicine disrupts the acquisition and performance of a working memory task in the radial arm maze. Neurotoxicology 9, 521-528.

Miller, S., Yasuda, M., Coats, J. K., Jones, Y., Martone, M. E., and Mayford, M. (2002). Disruption of dendritic translation of CaMKIIalpha impairs stabilization of synaptic plasticity and memory consolidation. Neuron $36,507-519$.

Ming, G. L., and Song, H. (2005). Adult neurogenesis in the mammalian central nervous system. Annu. Rev. Neurosci. 28, 223-250.

Morgan,J.I.,Cohen,D.R.,Hempstead, J. L., and Curran, T. (1987). Mapping patterns of c-fos expression in the central nervous system after seizure. Science 237, 192-197.

Nakazawa, K., Sun, L. D., Quirk, M. C., Rondi-Reig, L., Wilson, M. A., and Tonegawa, S. (2003). Hippocampal CA3 NMDA receptors are crucial for memory acquisition of one-time experience. Neuron 38, 305-315.

Nyffeler, M., Yee, B. K., Feldon, J., and Knuesel, I. (2008). Abnormal differentiation of newborn granule cells in age-related working memory impairments. Neurobiol. Aging. [Epub ahead of print].

Olton, D. S., Walker, J. A., and Wolf, W. A (1982). A disconnection analysis of hippocampal function. Brain Res. 233, 241-253.

Oyoshi, T., Nishijo, H., Asakura, T., Takamura, Y., and Ono, T. (1996). Emotional and behavioral correlates of mediodorsal thalamic neurons during associative learning in rats. I. Neurosci. 16, 5812-5829.

Reijmers, L. G., Perkins, B. L., Matsuo, N., and Mayford, M. (2007). Localization of a stable neural correlate of associative memory. Science 317, 1230-1233.

Schwegler, H., Crusio, W. E., and Brust, I. (1990).Hippocampal mossy fibers and radial-maze learning in the mouse: a correlation with spatial working memory but not with non-spatial reference memory. Neuroscience 34, 293-298.

Silva, A. J., Paylor, R., Wehner, J. M., and Tonegawa, S. (1992). Impaired spatial learning in alpha-calcium-calmodulin kinase II mutant mice. Science 257, 206-211.

Silva, A. J., Rosahl, T. W., Chapman, P. F. Marowitz, Z., Friedman, E., Frankland, P.W., Cestari, V., Cioffi, D., Sudhof, T. C., and Bourtchuladze, R. (1996). Impaired learning in mice with abnormal short-lived plasticity. Curr. Biol. 6, 1509-1518. 
Smeyne, R. J., Schilling, K., Robertson, L., Luk, D., Oberdick, J., Curran, T., and Morgan,J.I.(1992).fos-lacZ transgenic mice: mapping sites of gene induction in the central nervous system. Neuron $8,13-23$.

Thierry, A. M., Gioanni, Y., Degenetais, E., and Glowinski,J.(2000).Hippocampoprefrontal cortex pathway: anatomical and electrophysiological characteristics. Hippocampus 10, 411-419.

Torrey, E. F., and Peterson, M. R. (1974). Schizophrenia and the limbic system. Lancet 2, 942-946.

Touzani, K., Marighetto, A., and Jaffard, R. (2003). Fos imaging reveals ageingrelated changes in hippocampal response to radial maze discrimination testing in mice. Eur. J. Neurosci. 17, 628-640.

Vann, S. D., Brown, M. W., Erichsen, J. T., and Aggleton, J. P. (2000). Fos imaging reveals differential patterns of hippocampal and parahippocampal subfield activation in rats in response to different spatial memory tests. J. Neurosci. 20, 2711-2718.

Walsh, T. J., Schulz, D. W., Tilson, H. A., and Schmechel, D. E. (1986). Colchicine-induced granule cell loss in rat hippocampus: selective behavioral and histological alterations. Brain Res. 398, 23-36.

Wang, H., Shimizu, E., Tang, Y. P., Cho, M., Kyin, M., Zuo, W., Robinson, D. A., Alaimo, P. J., Zhang, C., Morimoto, H., Zhuo, M., Feng, R., Shokat, K. M., and Tsien, J. Z. (2003). Inducible protein knockout reveals temporal requirement of CaMKII reactivation for memory consolidation in the brain. Proc. Natl. Acad. Sci. U.S.A. 100, 4287-4292.

Wang, K. H., Majewska, A., Schummers, J., Farley, B., Hu, C., Sur, M., and Tonegawa, S. (2006). In vivo two-photon imaging reveals a role of arc in enhancing orientation specificity in visual cortex. Cell 126, 389-402.
Weinberger, D. R. (1988). Schizophrenia and the frontal lobe. Trends Neurosci. 11, 367-370.

Weinberger, D. R. (1996). On the plausibility of "the neurodevelopmental hypothesis" of schizophrenia. Neuropsychopharmacology 14, 1S-11S.

Wilson, Y., Nag, N., Davern, P. Oldfield, B. J., McKinley, M. J., Greferath, U., and Murphy, M. (2002). Visualization of functionally activated circuitry in the brain. Proc. Natl. Acad. Sci. U.S.A. 99, 3252-3257.

Yamasaki, N., Maekawa, M., Kobayashi, K., Kajii, Y., Maeda, J., Soma, M., Takao, K., Tanda, K., Ohira, K., Toyama, K., Kanzaki, K., Fukunaga, K., Sudo, Y., Ichinose, H., Ikeda, M., Iwata, N., Ozaki, N., Suzuki, H., Higuchi, M., Suhara, T., Yuasa, S., and Miyakawa, T. (2008). Alpha-CaMKII deficiency causes immature dentate gyrus, a novel candidate endophenotype of psychiatric disorders. Mol. Brain 1, 6.
Conflict of Interest Statement: The authors declare that the research was conducted in the absence of any commercial or financial relationships that could be construed as a potential conflict of interest.

Received: 11 May 2009; paper pending published: 29 June 2009; accepted: 11 August 2009; published online: 02 September 2009. Citation: Matsuo N, Yamasaki N, Ohira K, Takao K, Toyama K, Eguchi M, Yamaguchi $S$ and Miyakawa T (2009) Neural activity changes underlying the working memory deficit in alpha-CaMKII heterozygous knockout mice. Front. Behav. Neurosci. 3:20. doi: 10.3389/neuro.08.020.2009 Copyright (c) 2009 Matsuo, Yamasaki, Ohira, Takao, Toyama, Eguchi, Yamaguchi and Miyakawa. This is an open-access article subject to an exclusive license agreement between the authors and the Frontiers Research Foundation, which permits unrestricted use, distribution, and reproduction in any medium, provided the original authors and source are credited. 